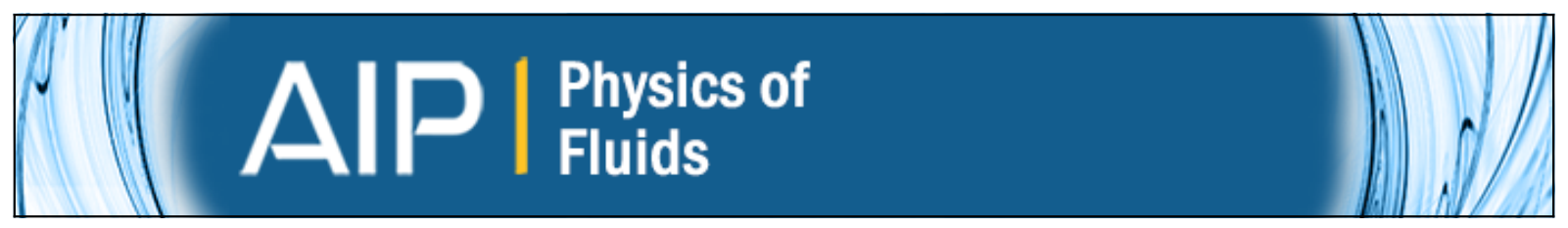

\title{
Numerical investigation of pulsatile flow in endovascular stents
}

A. Rouhi, U. Piomelli, and P. Vlachos

Citation: Physics of Fluids (1994-present) 25, 091905 (2013); doi: 10.1063/1.4821618

View online: http://dx.doi.org/10.1063/1.4821618

View Table of Contents: http://scitation.aip.org/content/aip/journal/pof2/25/9?ver=pdfcov

Published by the AIP Publishing

Copyright by the American Institute of Physics. Numerical investigation of pulsatile flow in endovascular stents. Rouhi, A. and Piomelli, U. and Vlachos, P., Physics of Fluids (1994present), 25, 091905 (2013), DOI:http:// dx.doi.org/10.1063/1.4821618

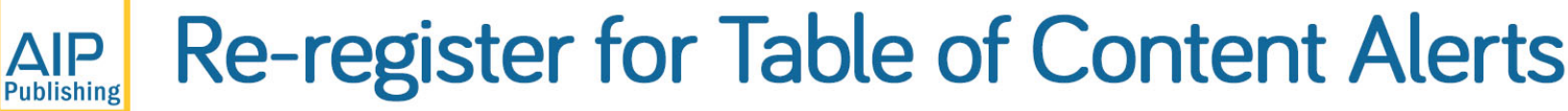




\title{
Numerical investigation of pulsatile flow in endovascular stents
}

\author{
A. Rouhi, ${ }^{1}$ U. Piomelli, ${ }^{1, a)}$ and P. Vlachos ${ }^{2}$ \\ ${ }^{1}$ Department of Mechanical and Materials Engineering, Queen's University, \\ Kingston, Ontario K7L 3N6, Canada \\ ${ }^{2}$ Department of Mechanical Engineering, Virginia Tech, 114U Randolph Hall, \\ Blacksburg, Virginia 24061, USA
}

(Received 9 March 2013; accepted 20 August 2013; published online 27 September 2013)

The flow in a plane channel with two idealized stents (one $\Lambda$-shaped, the other $X$-shaped) is studied numerically. A periodic pressure gradient corresponding to one measured in the left anterior descending coronary artery was used to drive the flow. Two Reynolds numbers were examined, one $(R e=80)$ corresponding to resting conditions, the other $(R e=200)$ to exercise. The stents were implemented by an immersed boundary method. The formation and migration of vortices that had been observed experimentally was also seen here. In the previous studies, the compliance mismatch between stent and vessel was conjectured to be the reason for this phenomenon. However, in the present study we demonstrate that the vortices form despite the fact that the walls were rigid. Flow visualization and quantitative analysis lead us to conclude that this process is due to the stent wires that generate small localized recirculation regions that, when they interact with the near-wall flow reversal, result in the formation of these vortical structures. The recirculation regions grow and merge when the imposed waveform produces near-wall flow reversal, forming coherent quasi-spanwise vortices, that migrate away from the wall. The flow behavior due to the stents was compared with an unstented channel. The geometric characteristics of the $\Lambda$-stent caused less deviation of the flow from an unstented channel than the $X$-stent. Investigating the role of advection and diffusion indicated that at $R e=80$ advection has negligible contribution in the transport mechanism. Advection plays a role in the generation of streamwise vortices created for both stents at both Reynolds numbers. The effect of these vortices on the near-wall flow behavior is more significant for the $\Lambda$-stent compared to the $X$-stent and at $R e=200$ with respect to $R e=80$. Finally, it was observed that increasing the Reynolds number leads to early vortex formation and the creation of the vortex in a stented channel is coincident with the near wall flow reversal in an unstented one. (c) 2013 AIP Publishing LLC. [http://dx.doi.org/10.1063/1.4821618]

\section{INTRODUCTION}

In a healthy blood vessel, fatty tissues may accumulate on the inner vessel wall, depending on such factors as diet, daily activity, age, genetic predisposition, etc. This may lead to the narrowing of the blood vessel and, eventually, to flow blockage (Hajjar and Nicholson ${ }^{1}$ ). Stents are wired metallic tubes that are implanted where there is abnormal narrowing of the vessel wall; they widen the vessel lumen and restore the blood flow. Although stents are widely used in interventional cardiology, due to the minimally invasive character of their implantation (compared with the alternative, which in most cases is bypass surgery), their beneficial effect may be only temporary, since re-accumulation of fatty tissues and re-narrowing of the vessel may happen. This phenomenon, known as re-stenosis

\footnotetext{
a)Electronic mail: ugo@me.queensu.ca
} 
(Chen et $a .^{2}$ ), may occur within six months of stent implantation, mainly due to fluid dynamical effects (Murphy and Boyle ${ }^{3}$ ).

In a blood vessel, transport of fatty tissues and other biological molecules toward the vessel wall occurs due to diffusive and advective processes in the blood flow (Bluestein $e t$ al. ${ }^{4}$ and Caro et $a .^{5}{ }^{5}$. As fatty cells approach the wall, the shear stress induced by the flow determines whether or not these molecules will adhere to the vessel wall (Fry, ${ }^{6}$ Caro et al.,${ }^{5}$ and Zarins et al. ${ }^{7}$ ). Malek et $a l^{8}{ }^{8}$ observed that regions of low wall shear-stress are the regions with high risk of fatty-tissue accumulation.

While implantation of the stent prevents the vessel narrowing, its presence significantly changes the flow patterns. Regions with low wall shear-stress and flow recirculation may appear, causing the biological molecules to accumulate; considering the change in the near wall flow behavior and wall shear-stress distribution, several stent shapes have been proposed that, by altering the flow near the stent, attempt to decrease the occurrence of re-stenosis. Both experimental and computational studies have investigated this problem. The early studies attempted to correlate the risk of re-stenosis to the fluid mechanical parameters, while subsequent experimental and computational investigations examined the critical parameters and locations with high risk of re-stenosis for different geometries and flow conditions.

Wentzel et al. ${ }^{9}$ related the dynamics of the flow to the thickness of fatty tissues using experiments and numerical studies; the geometry was a curved vessel reconstructed by an ultra-sound technique. A steady velocity profile was applied, obtained from a previous calculation of a curved pipe flow. The results revealed that a higher thickness of the internal wall is correlated with the regions with low wall shear-stress. However, in this study the flow field was not studied in detail and only the shear stress was considered. Duraiswamy et al..$^{10}$ extended this study of flow behavior and adhesion of biological molecules, performing both experiments and computations. A two-dimensional (2D) channel flow implanted with 2D stents was studied under pulsatile flow condition. The particles studied were adhesive cells (platelets) obtained from the blood of human volunteers, labeled by fluorescent dye. Computational Fluid Dynamics (CFD) was also used: a simulation was performed using the finite-volume solver CFD-ACE (CFD Research Corporation, Huntsville, AL) with the same geometry and flow condition used in experiment to find the particle trajectories. Comparing the streamlines obtained from CFD simulation and the concentration of platelets revealed that the recirculation zones created upstream and downstream of the stent struts are locations where high concentration of platelets were observed, probably due to the advection of platelets toward the wall in these regions. The study was performed in steady conditions corresponding to the maximum and minimum flow rates. No investigation was carried out of the effects of flow acceleration. However, Yazdani et al. ${ }^{12}$ and Charonko et al. ${ }^{13,14}$ observed, in their experimental studies, that during the acceleration and deceleration phases the recirculation zones are highly disturbed.

Duraiswamy et al. ${ }^{11}$ extended the work by Duraiswamy et al. ${ }^{10}$ into realistic 3D models by repeating the same experiment using three real stent shapes. Comparison between the platelet depositions obtained from experiments using dye-marked platelets with the streamlines and the flow field calculated using CFD computations showed that the highest concentration of platelets occurs near stent connectors, where the flow streamline patterns were complex. Moreover, the regions where the flow is directed towards the wall have higher concentration of platelets than those where the flow is parallel to the wall. This caused the concentration of platelets to be higher downstream of each stent strut than upstream of it.

The correlations observed between the fluid mechanics and their clinical consequences provide a motivation for parametric investigations on the stent geometries and flow conditions. Berry et al. ${ }^{15}$ performed a numerical study of an idealized 2D geometry using waveforms with negative flowrates during part of the cycles; the inflow condition was a linear Couette-flow profile adjusted based on the imposed waveform; the flow domain extended only up to a specific height above the wall, to decrease the cost of the calculations. This type of inlet boundary condition, however, is not consistent with a pulsatile waveform, which can result in an inflection point in the velocity profile that affects the flow field $\left(\right.$ Moore $\left.^{16}\right)$. A parametric study was performed of the effect of stent thickness and inter-strut spacing on the size of the recirculation zones created upstream and downstream of the stent. 
LaDisa et al. ${ }^{17}$ used CFD to study the alterations in wall shear-stress distribution after placing a slotted-tube stent design with a steady parabolic inlet velocity profile corresponding to maximum and minimum flowrates for human conditions. This was the first computational study of stented vessels in which the 3D geometry of stents was considered. The risks of vessel wall-thickening were evaluated based on local wall shear-stress values. It was observed that the shear stress is maximum on the surface of the stent wires, and minimum on the vessel wall in the corners of each strut, where recirculation zones were created. Again, in this study the effects of acceleration, which may result in inflectional instabilities, were neglected, and the wall shear-stress was the only parameter considered.

This work was extended by LaDisa et al. ${ }^{18}$ by first considering the effect of the difference in the stent diameter with respect to the vessel diameter, and also changing the number of stent struts and the wire thickness. The inflow condition was a steady parabolic profile with a mean value corresponding to the time-averaged flowrate of a characteristic waveform for a human vessel. The results showed that several parameters increase the areas of low wall shear-stress, including increasing the ratio of vessel diameter to the stent diameter, increasing the number of stent struts, and increasing the stent height.

LaDisa et al. ${ }^{19}$ further increased the configuration complexity by considering the flattening of the vessel wall. When four stent struts in a cross section were used, the cross section after stent implantation was polygonal (instead of circular); when eight struts were used, however, the cross-section was almost circular. Following LaDisa et al. ${ }^{18}$ the difference between the effective stent diameter and that of the vessel was considered. An unsteady velocity profile was imposed at the inlet, and the flow was allowed to develop over an extended entrance length. The waveform gave positive flowrate throughout the cycle. As expected, the case with four struts differed more significantly from the circular cross section than the eight-strut one, producing larger areas of low wall shear-stress. The velocity profiles showed that no reversed flow occurred near the wall through the cycle, partly due to the waveform chosen; most waveforms obtained from humans, however, have periods of negative flowrate, which produces reversed flow near the wall which (depending on the magnitude of the negative flow-rate) may extend toward the channel centreline. ${ }^{20}$ LaDisa et al. ${ }^{21}$ added curvature of the blood vessel. Two cases were considered; one with the curved stent adapting to the shape of the vessel, the other with a rigid stent that straightens the vessel. The results revealed that the velocity profiles in a straightened vessel are slightly skewed toward the wall, and cause larger wall shear-stress in comparison with the curved ones. This caused the area of low wall shear-stress to be larger in curved vessels than in the straightened ones.

The wall shear-stress was the main parameter studied in all the previous CFD works. However, since the kinematics of the flow have a great impact on the near-wall flow behavior and distribution of wall shear-stress, it is necessary to study the behavior of the flow during a cycle in more detail. Also, the effect of the reversed flow that takes place during part of the cycle in human waveforms needs to be investigated, as well as the effect of acceleration and deceleration on the stability of the recirculation regions that have been observed by many researchers. Among the first to perform a study of this type were Yazdani et al. ${ }^{12}$ and Charonko et al. ${ }^{13,14}$ Yazdani et al. ${ }^{12}$ used DPIV (Digital Particle Image Velocimetry), and an imposed waveform including an acceleration and a deceleration phase. The most significant finding in that study was the observation of vortical structures during these two phases, and their migration to the center of the channel. The reason for the creation of these vortices was conjectured to be the difference in the distensibility of the stent with respect to the vessel wall and the pulsatile nature of the waveform; this difference in the distensibility is known as "compliance mismatch."

A similar experiment was conducted by Charonko et al. ${ }^{13}$ in which Particle Image Velocimetry (PIV) was used, and two waveforms were imposed (for exercise and resting conditions); both had negative flowrate in their cycles, but were different than the one used by Yazdani et al. ${ }^{12}$ Four commercially available stents were examined. The creation and migration of the vortices was observed during the cycle of the imposed waveform. Since PIV was used, only a plane cross-section could be observed, and their spatial topology could not be determined. Spatial and time averaged wall shear-stresses were studied for all stents. 
Charonko et al. ${ }^{14}$ concentrated on a single stent shape and studied the effect of the stent length, overlap between two stents, and expansion diameter of the stent. The wall shear-stress was studied for different configurations and it was observed that its value does not change (for different configurations) downstream of the first strut. Note that in Charonko et al. ${ }^{13}$ the major focus was on the kinematics of the flow while in their subsequent work ${ }^{14}$ the wall shear-stress was the main objective.

Despite the extensive work, several issues require further investigations. The vortical structure observed experimentally induces low wall shear-stress $;{ }^{12}$ their generation and development, therefore, need to be investigated. To the authors' knowledge, no previous CFD simulation in stented vessels was found in which the vortex dynamics were explored in detail and their 3D structure remains mostly unknown. The reason for the creation of vortices was conjectured to be the pulsatile nature of the waveform and compliance mismatch, ${ }^{12}$ but no definitive conclusion could be reached, based on the experimental data, on the relative importance of these effects. The vortex creation was observed for three waveforms that had negative flowrates during their cycles; the possibility of vortex creation even when there is no negative flow-rate in the waveform is another open question. Moreover, it is important to determine for physiological stented-artery flows whether the transport mechanism is governed by advection or diffusion.

To answer these questions, we studied the flow behavior for various waveforms, some of which are unidirectional, while most include reversed flow. The waveform used by Charonko et al. ${ }^{13}$ is used as a base case, and applied on a periodic channel in which an immersed boundary method (IBM) is used to model the stents. Two idealized geometries are created, inspired by two commercially available stents. The flow behavior is studied at two Reynolds numbers corresponding to resting and exercise conditions. The contribution of advection against diffusion to the transport mechanism is evaluated, the deviation of the flow from an unstented channel (which represents a healthy vessel) is examined at both Reynolds numbers. In the following, we will first describe the numerical model and the problem configuration. We will then present and validate the model, and present the results, both in terms of flow kinematics and dynamical parameters. Some conclusions and recommendations for future work will conclude the article.

\section{PROBLEM FORMULATION}

In this study, we consider the incompressible flow of a Newtonian fluid. Although nonNewtonian behavior can be expected if the shear rate is less than $100 \mathrm{~s}^{-1}$ (Seo et al. ${ }^{25}$ ) this effect is not considered here; furthermore, the framework of this study is based on the work done by Charonko et al. ${ }^{13,14}$ in which the working fluid was a mixture of water and glycerine approximating the density and viscosity of human blood. Gravity is neglected in this problem as it has minor effect in comparison with pressure and viscous forces. ${ }^{22,23}$ The governing equations for an incompressible, unsteady, viscous flow for a Newtonian fluid are

$$
\begin{gathered}
\frac{\partial u_{i}}{\partial x_{i}}=0, \\
\frac{\partial u_{i}}{\partial t}+\frac{\partial}{\partial x_{j}}\left(u_{i} u_{j}\right)=-\frac{\partial p}{\partial x_{i}}+\frac{1}{R e} \frac{\partial^{2} u_{i}}{\partial x_{j} \partial x_{j}}+\frac{\overline{d P}}{d x} \delta_{1 i},
\end{gathered}
$$

where $u_{i}$ are the velocities $\left(u_{1}, u_{2}\right.$, and $u_{3}$, or $u, v$, and $w$, are the velocity components in the streamwise, wall-normal, and spanwise directions $x, y$, and $z$, respectively), $p$ is the pressure (divided by the density $\rho$, which we assume to be unity), $t$ is time, and $R e$ is Reynolds number based on appropriate velocity and length scales. No-slip and symmetry boundary conditions are applied at the lower wall and top boundary, respectively. Periodic boundary conditions are used in the streamwise and spanwise directions. The velocity components and pressure are normalized by $U_{b}$ and $\rho U_{b}^{2}$, respectively, where $U_{b}$ is the time-averaged bulk velocity during one period of the waveform; $t$ is non-dimensionalized by $H / U_{b}$ where $H$ is the channel half-height. The Reynolds number is defined as $R e=U_{b} H / v$ where $v$ is the kinematic viscosity of the fluid. 

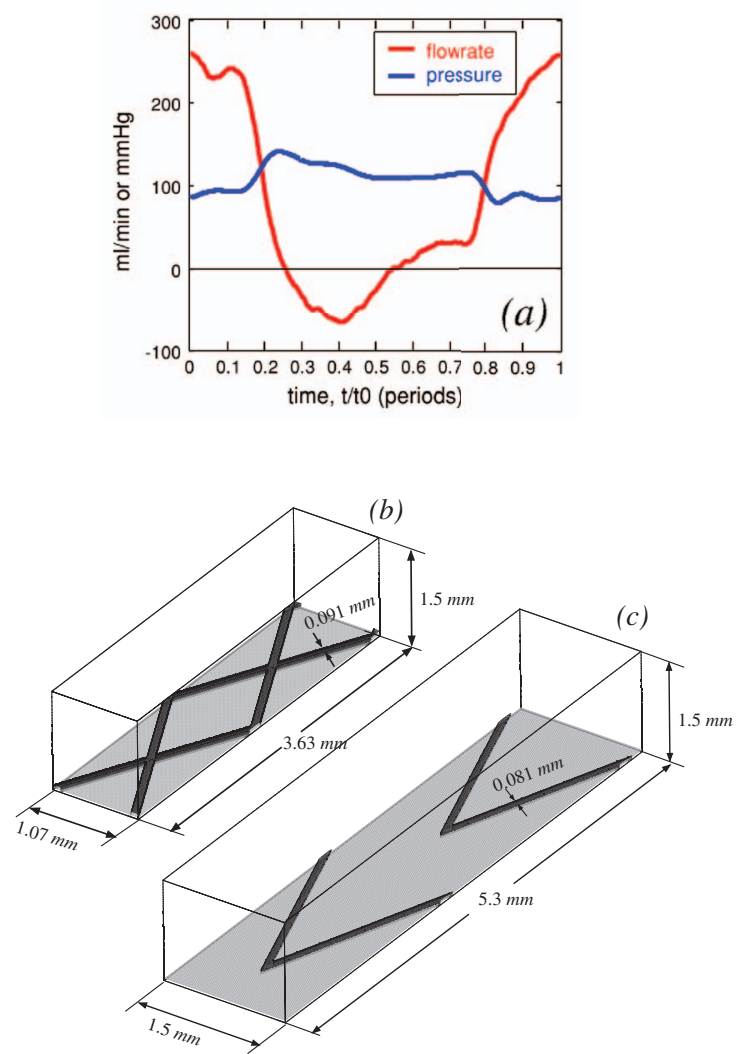

FIG. 1. (a) Velocity and pressure waveforms. ${ }^{13,14}$ (b) $X$-stent geometry. (c) $\Lambda$-stent geometry.

An unsteady mean-pressure gradient $(\overline{d P / d x})$ is added to the $x$-momentum equation to drive the flow and provide the desired flowrate at each time-step. The waveform imposed is based on the resting condition in the left anterior descending (LAD) coronary artery used by Charonko et al. ${ }^{13,14}$ (Figure 1(a)). For exercise conditions, the same waveform was used but the Reynolds number was increased. The period of the oscillations were $T_{c}=0.87 \mathrm{~s}$ for resting condition and $T_{c}=0.348 \mathrm{~s}$ for exercise condition, equivalent to 69 and 172 beats per minute, respectively.

A well-validated finite-difference code (Keating et $a l .{ }^{24}$ ) based on a staggered grid was used to solve the discretized equations. The governing equations are integrated in time using a fractionalstep method. ${ }^{26,27}$ Second-order central differencing was used for all spatial gradients. The time advancement was a hybrid method: a three-step, second-order-accurate, Runge-Kutta scheme was used for all terms of the momentum equation except the wall-normal diffusion term, for which the second-order Crank-Nicolson scheme was used. The 3D Poisson equation was reduced by Fourier transforms in the streamwise and spanwise directions to a series of 1D Helmholtz equations in wave-number space, which was then solved by a direct method. The code is parallelized using the Message-Passing Interface (MPI) protocol.

The stents are implemented by an immersed boundary method based on the volume-of-fluid (VOF) approach. ${ }^{28} \mathrm{~A}$ force term is introduced on the right-hand-side of the momentum equations to reduce the velocity inside the solid object (the stent) depending on the fraction of the solid object in each grid cell. Therefore, the fractional step method is modified into (i) predicting the intermediate velocity field using convection and diffusion terms, (ii) multiplying the predicted velocity by the volume-of-fluid to account for the effect of stents, (iii) solving the Poisson equation based on the new predicted velocity field, and (iv) adding the pressure force term to correct the velocity field and make it divergence free. Using this method, two idealized geometries inspired by two commercially available stents (Endeavor ${ }^{\circledR}$ and XIENCE_V ${ }^{\circledR}$ stents produced by Medtronic ${ }^{\complement}$ and Abbott Vascular ${ }^{\odot}$, respectively) are created; they are shown in Figure 1 and will be referred to as the 


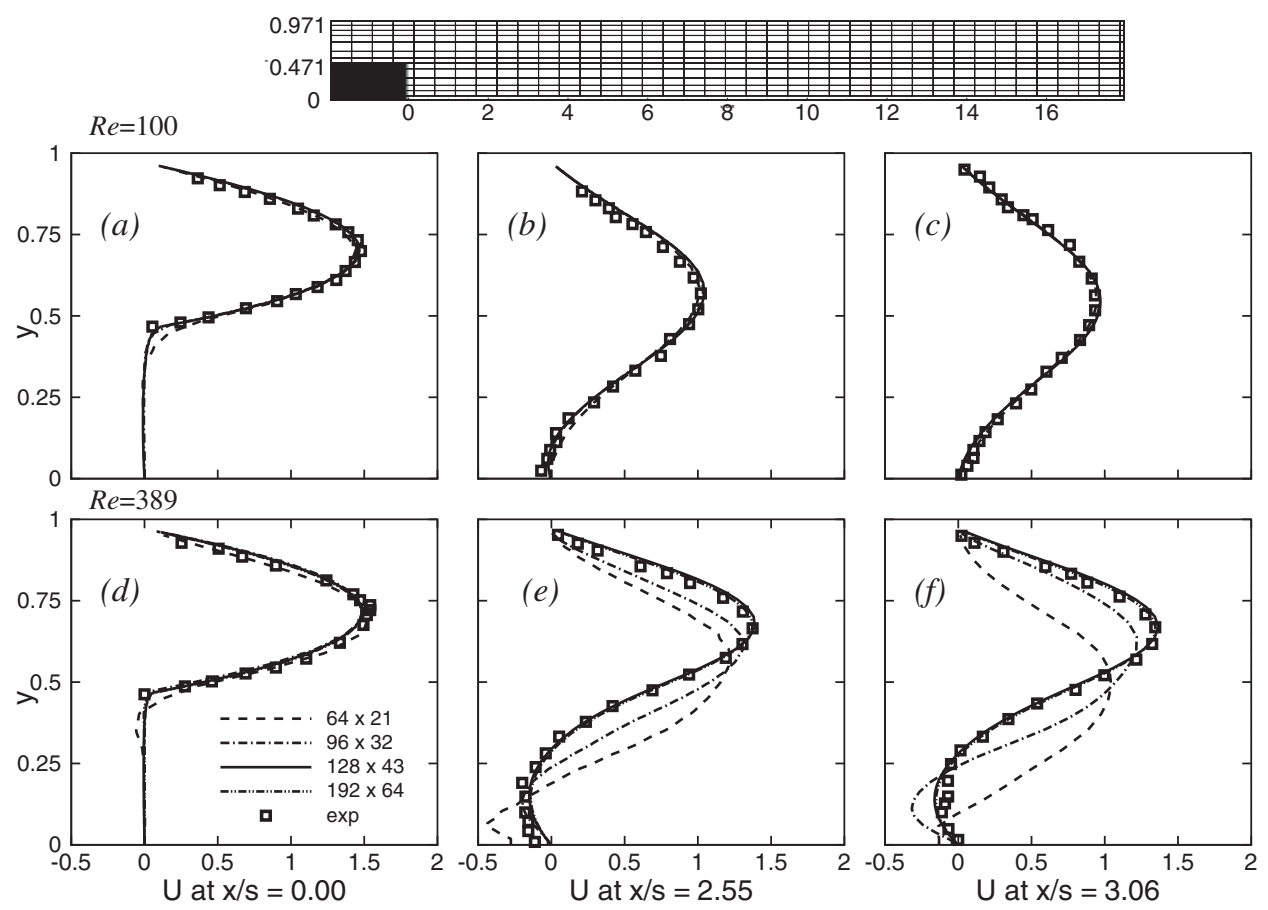

FIG. 2. Comparison of the velocity profiles downstream of a backward-facing step ( $\mathrm{s}$ is the step height) using the IBM (lines) and the experimental measurements by Armaly $e t a l .{ }^{30}$ (symbols). (a)-(c) are at $R e=100$ and (d)-(f) are at $R e=389$.

$X$ - and $\Lambda$-stents, respectively. The $X$-stent is closely similar to its real counterpart, while the $\Lambda$-stent does not include connectors and curved parts of the struts that are replaced with straight segments. All the dimensions were assigned based on their clinical counterparts. Note that the $\Lambda$-stent has a preferential direction, while the $X$-stent presents the same geometry to the flow during forward or reversed flow. Moreover, that portion of the vessel-wall area exposed to the flow is smaller for the $X$-stent than for the $\Lambda$-stent. The computational domain included two elements in the streamwise direction, and one in the spanwise direction.

Validation of the IBM was performed extensively. First, we carried out calculations of the flow over a backward-facing step and compared the results with the experiments of Armaly et al. ${ }^{30}$ (Figure 2). At low Reynolds number ( $R e=100$ based on twice the height of the inlet, small channel and the mean velocity at the inlet), we obtained excellent agreement with the experiments when 96 points were used in the streamwise direction and 32 in the wall-normal one. At $R e=389,128 \times 43$ points were required to achieve grid-converged results. The grids used in the present calculations, as discussed below, resolve the obstacle in greater detail than those used for this validation.

In a second test, periodic obstacles were created using both IBM for the staggered-grid code and body conforming grid using OpenFOAM ${ }^{\circledR 29}$ (an unstructured, open source solver). Figure 3(a) shows the geometry configuration and Figure 3(b) shows the location of data extraction, which is behind the obstacle in the wake region where the flow circulation occurs. The intention for the angled obstacles was to simulate the angled orientation of the stent wires with respect to the main flow direction. The Reynolds number in this study was set to $R e=150$ to reproduce the flow condition in blood vessels. The velocity component normal to the obstacle was compared at different grid resolutions using IBM setup in the staggered-grid code with OpenFOAM (Figure 3(c)). These grid resolutions used in each solver along with the corresponding number of cells on each edge of the stent wire are summarized in Table I. Considering Figure 3(c) with Table I indicates that to achieve convergence using the immersed boundary method, 14 cells on each edge of the stent wire are required, whereas the use of a body fitted grid reduces this number to 8. Although using 

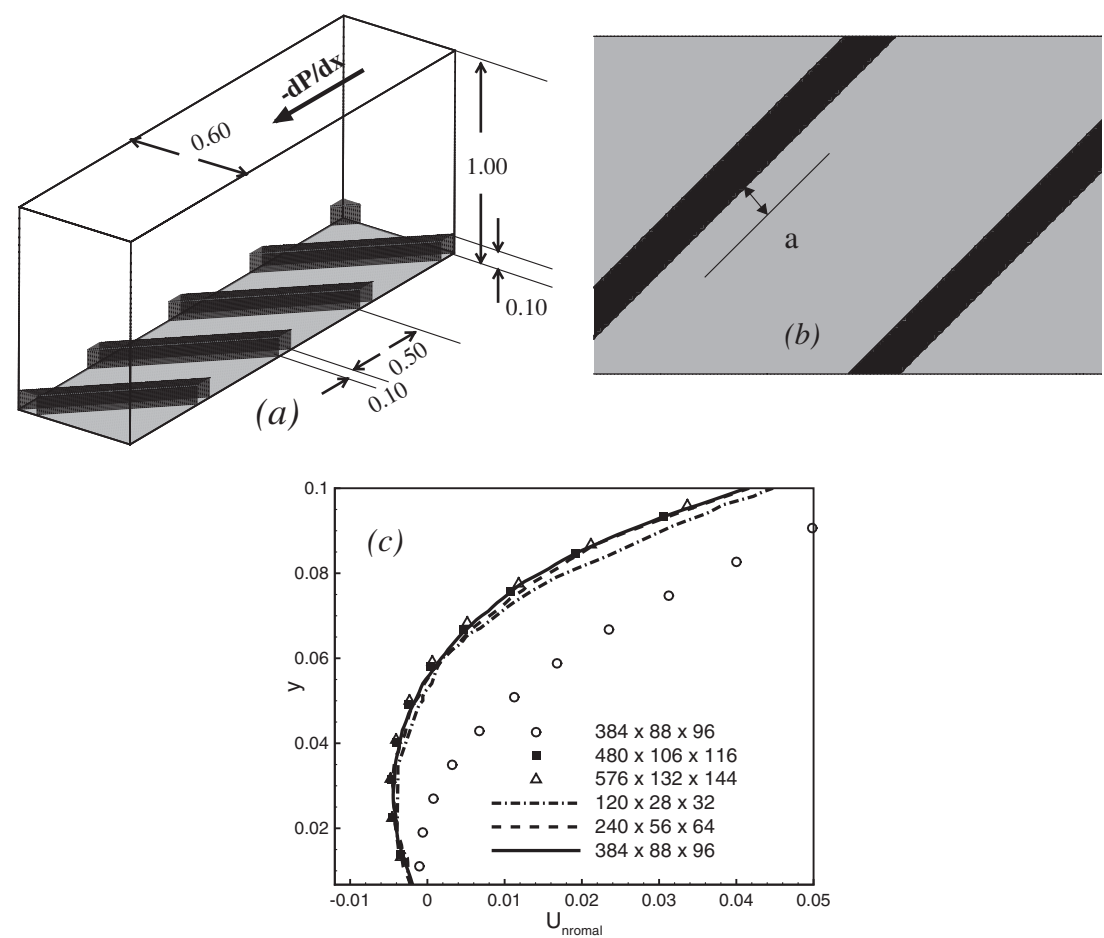

FIG. 3. Geometry configuration and size for simulation of periodic angled obstacles (a). Location of data extraction (b) and comparison of velocity profiles (normal component) at the corresponding location (c). Lines are the simulations performed using OpenFOAM and the symbols correspond to the IBM implemented in the staggered-grid code.

body-conforming grid with OpenFOAM requires a coarser grid resolution, the staggered-grid code with IBM has a lower computational cost by approximately a factor of 30 .

A grid convergence study was performed under both steady and unsteady cases at $R e=150$ for the $X$-stent; it was found that, to resolve the flow, 16 grid points were needed on each side of the stent wire (resulting in $256 \times 192 \times 192$ and $384 \times 256 \times 256$ grid points for $X$ - and $\Lambda$-stents, respectively). We also validated the use of a flat surface (as opposed to the axisymmetric one in the real flow) by comparing the results of the present model to one in a cylindrical geometry (which was solved using OpenFOAM). The results of this comparison are shown in Figure 4, and show that assuming flat surface for the vessel wall adds negligible error to the results. This is due to the large ratio between pipe diameter (or channel half-height) and stent height, 18:1.

Finally, in Figure 5, we compare the results obtained from the present simulations with those from experiment ${ }^{13,14}$ under the same flow conditions. The wall shear-stress, $\tau_{w}$, illustrated in the

TABLE I. Grid resolutions using IBM and OpenFOAM for the angled obstacle. The converged resolution is highlighted for each solver.

\begin{tabular}{lccr}
\hline \hline & IBM & & OpenFOAM \\
\cline { 3 - 4 } Grid & Cells & Grid & Cells \\
\hline $384 \times 88 \times 96$ & 11 & $120 \times 28 \times 32$ & 4 \\
$\mathbf{4 8 0} \times \mathbf{1 0 6} \times \mathbf{1 1 6}$ & $\mathbf{1 4}$ & $\mathbf{2 4 0} \times \mathbf{5 6} \times \mathbf{6 4}$ & $\mathbf{8}$ \\
$\mathbf{5 7 6} \times 132 \times 144$ & 17 & $384 \times 88 \times 96$ & 11 \\
\hline \hline
\end{tabular}



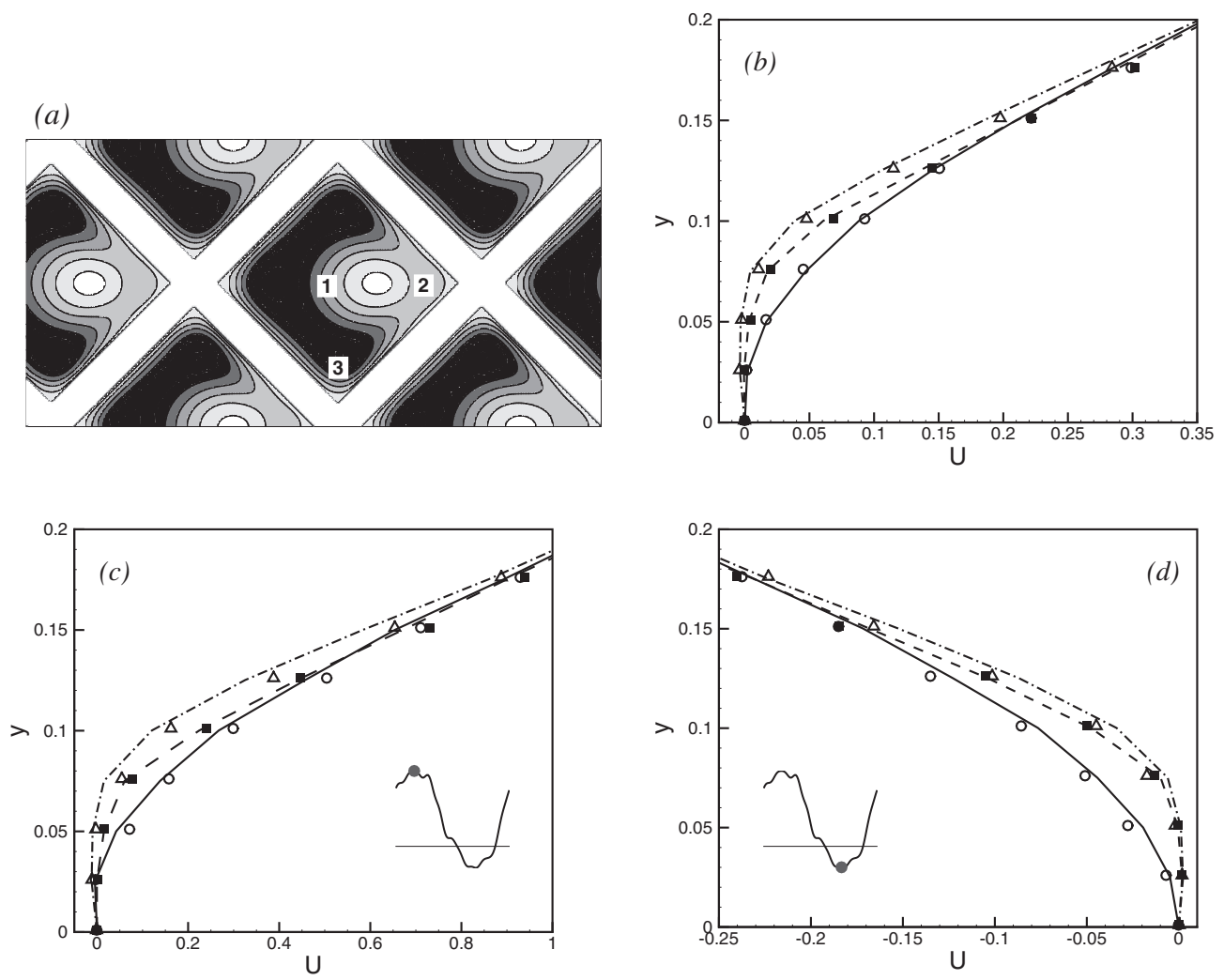

FIG. 4. Comparison of velocity profiles for planar case $(-,-\cdot-,---)$ and cylindrical case $(\circ, \mathbf{\square}, \triangle)$ at points $1(-, \circ), 2(---, \mathbf{\square})$, and $3(-\cdot-, \triangle)$. (a) Location of data extraction for comparison. (b) Comparison under steady flow. (c) and (d) Comparison under unsteady flow at the times indicated on the waveform.

figure, is defined as

$$
\tau_{w}(x, z, t)=\mu \times \operatorname{sgn}\left(\frac{\partial u}{\partial y}\right)\left[\left(\frac{\partial u}{\partial y}\right)^{2}+\left(\frac{\partial w}{\partial y}\right)^{2}\right]^{1 / 2}
$$

and is averaged over the entire wall. The agreement is acceptable, but the geometric details (especially the wetted area of the channel are important for the quantitative agreement of the results). In fact, a modified stent geometry (including connectors between the wires), gave better agreement with the real geometry. The agreement level for the idealized stents provides sufficient validation of the current model.
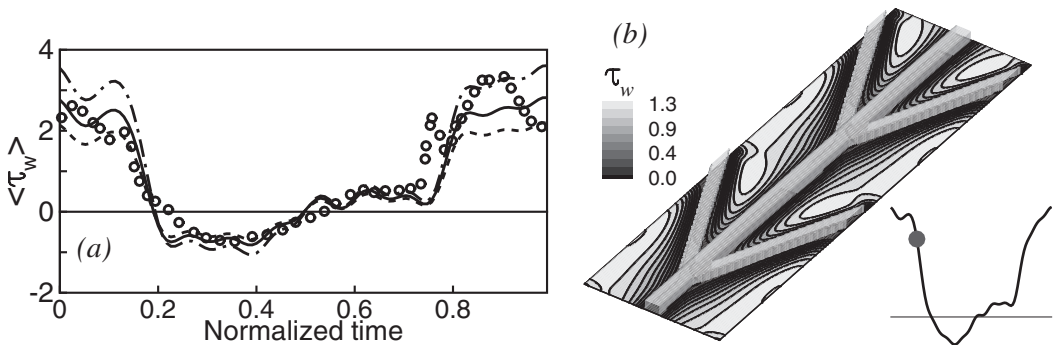

FIG. 5. (a) Comparison of spatially averaged wall shear-stress (in Pa). $\circ$ : experimental data ${ }^{14}$ for the XIENCE_V stent; - - - : $\Lambda$-stent; - - - : $X$-stent; —— modified $\Lambda$-stent. (b) Modified $\Lambda$-stent and wall shear-stress contours (in Pa). 


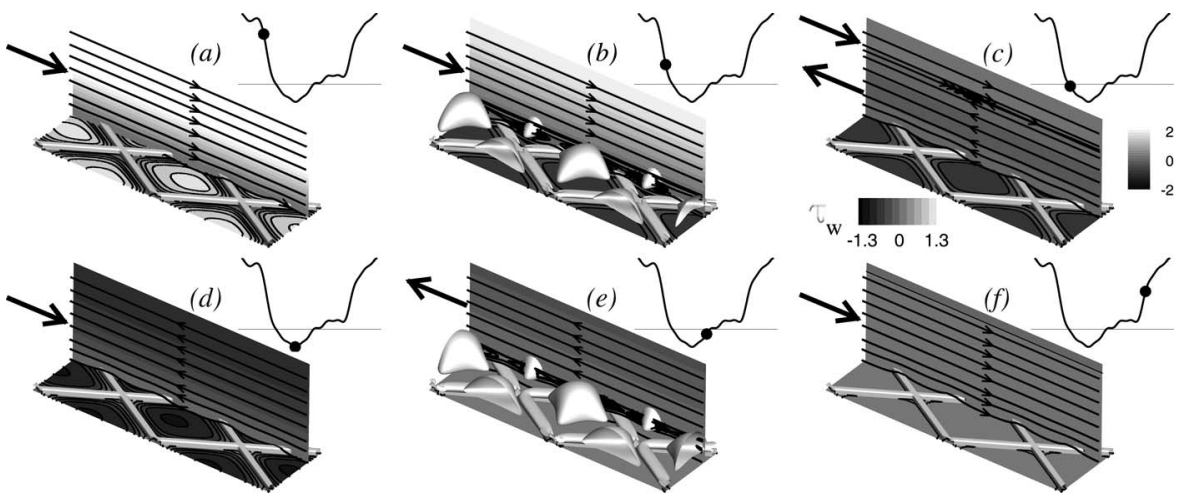

FIG. 6. (a)-(f) Flow visualization at six phases shown in the waveform inset; the mean-flow direction is indicated by arrow. $R e=80, X$-stent. Wall shear-stress $\left(\tau_{w}\right)$ is in Pa.

\section{RESULTS}

In the following, first the flow behavior is described at different times of the driving waveform for the $X$ - and $\Lambda$-stents. The importance of these vortical structures lies in their effect on the generation of low wall shear-stress regions. The presence of the stent leads to the creation of recirculation zones or large vortical structures, and the deviation of the flow from its normal behavior in a healthy vessel. This deviation is studied quantitatively next, by comparing the changes in the wall shearstress and velocity profiles that occur after stent placement, for the different stents. Then, the role of the Reynolds number in the transport mechanism is studied, and the significance of advection with respect to diffusion at the two Reynolds numbers is quantified. Finally, we examine the causes of the vortex generation by modifying the driving waveform to decrease or remove the flow reversal associated with the baseline waveform chosen.

Two major flow conditions are studied in this work. One is $R e=80$ corresponding to resting condition and the other one is at $R e=200$ related to the exercise condition. All the results are presented at both flow conditions and are compared.

\section{A. Flow description}

Figures 6 and 7 show six phases of the flow during one cycle. The vortical structures are identified using iso-surfaces of the second invariant of the velocity gradient tensor, $Q$

$$
Q=-\frac{1}{2} \frac{\partial u_{i}}{\partial x_{j}} \frac{\partial u_{j}}{\partial x_{i}}
$$
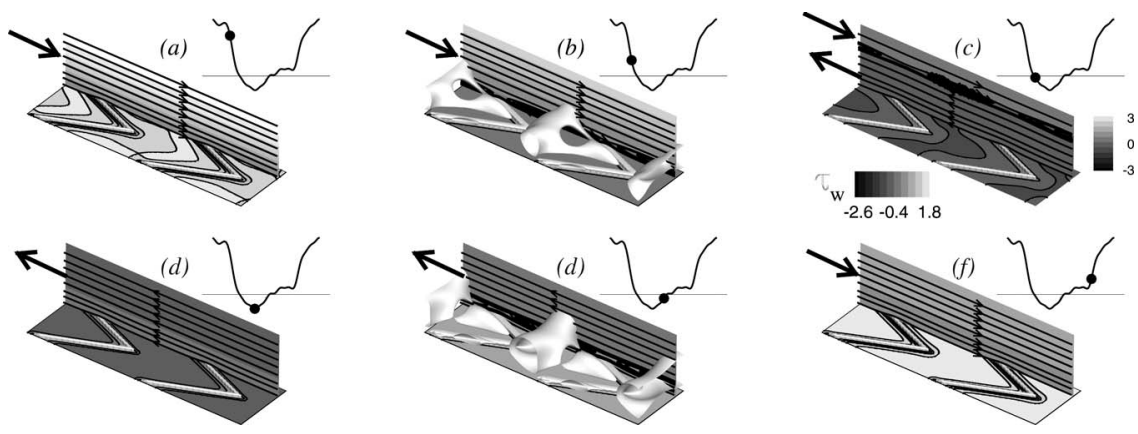

FIG. 7. (a)-(f) Flow visualization at six phases shown in the waveform inset; the mean-flow direction is indicated by arrow. $R e=80, \Lambda$-stent. Wall shear-stress $\left(\tau_{w}\right)$ is in Pa. 


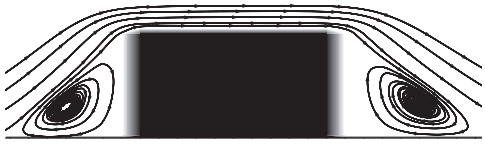

(a)

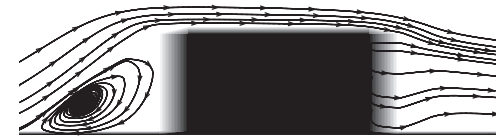

(c)

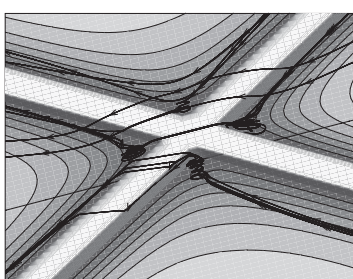

(b)

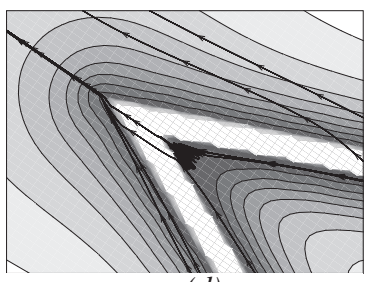

(d)

FIG. 8. Visualization of the recirculation zones around the stent struts $t / T_{c}=0.17$. (a) and (b): $X$-stent; (c) and (d) $\Lambda$-stent. The streamlines that are appearing downstream of the stent in part (d) are due to the convergence of the streamlines downstream of the vertex of the $\Lambda$-stent.

During the initial phases of the cycle (Figures 6(a) and 7(a)), when the flowrate is high, the flow separates downstream (due to the sharp corner) and upstream (due to the adverse pressure gradient) of the stent wires. A recirculation zone is formed upstream of the wire for both stent geometries (Figure 8). Immediately downstream of the wire, a recirculation zone is formed for the $X$-stent, but not for the $\Lambda$-stent. This is due to the motion of the fluid along the stent strut (Figure 8(d)), which displaces the separation streamline upwards, and prevents it from re-attaching to the wall. In the $X$-stent, the closed cavity formed between the struts prevents this phenomenon.

As the bulk flow decelerates, the recirculation zones created around the stent struts grow, merge, and form vortical structures between the struts (Figures 6(b) and 7(b)); this process of vortex formation is illustrated in Figure 9. As flow reversal starts, the extension of the recirculation zones increases until they occupy the whole region between consecutive struts. This process occurs in less than $0.01 T_{c}$. The reversed flow below the vortex causes vortex lift-off as the magnitude of negative flowrate increases (Figures 6(c) and 7(c)). The process of vortex formation, migration, and disappearance is repeated when the driving flowrate accelerates from a negative to a positive value

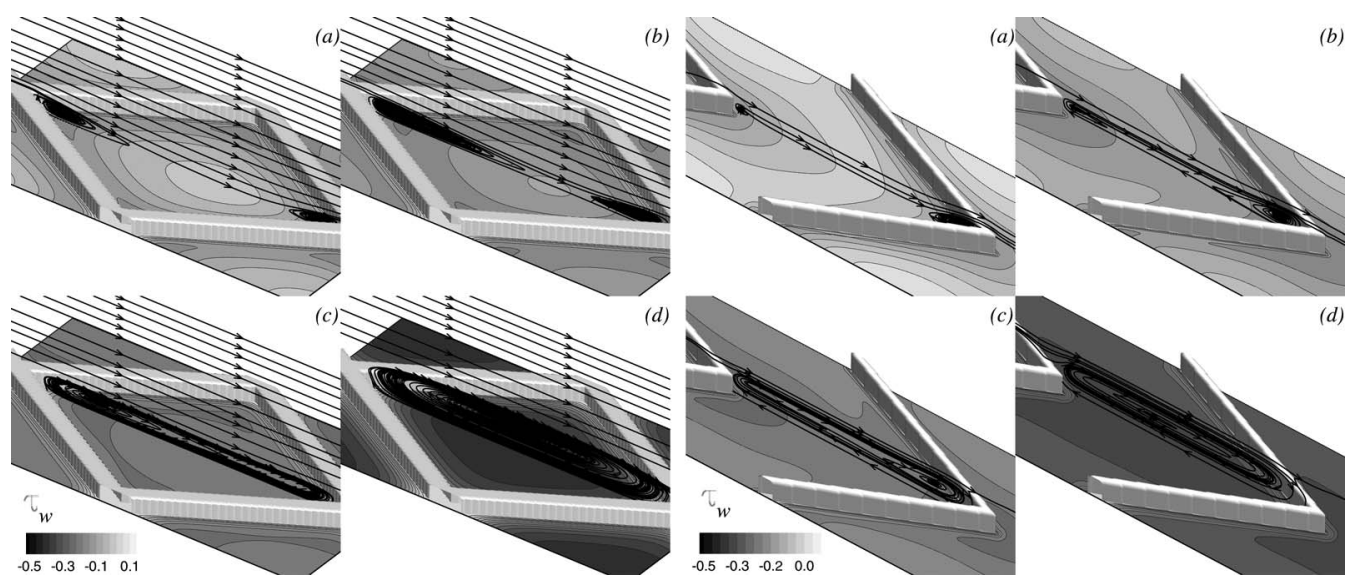

FIG. 9. Creation of the vortex during the flow reversal in the $X$ - (left) and $\Lambda$-stents. (a) $t / T_{c}=0.192$, (b) $t / T_{c}=0.196$, (c) $t / T_{c}=0.197$, and (d) $t / T_{c}=0.200$. Wall shear-stress $\left(\tau_{w}\right)$ is in Pa. 

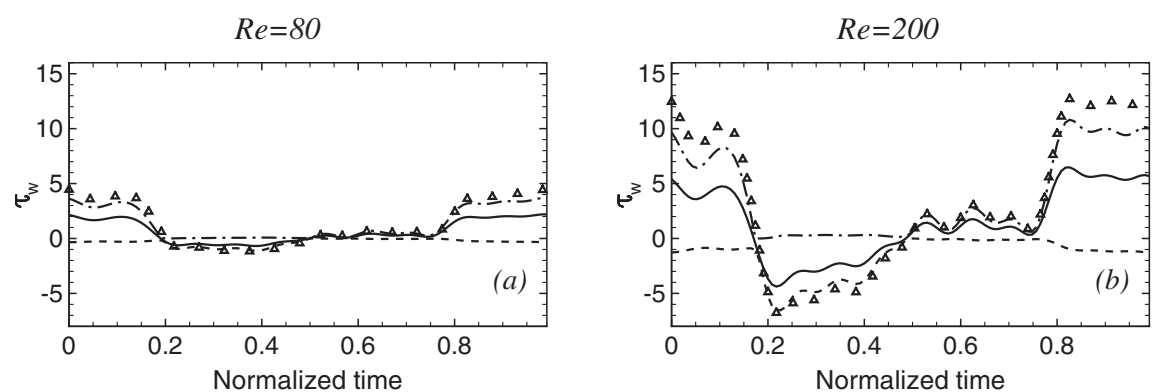

FIG. 10. (a) and (b) History of spatial-averaged (-), maximum (-- - ), and minimum (-- ) wall shear-stress $\left(\tau_{w}\right)$ and their comparison with wall shear-stress in an unstented channel $(\Delta)$ for the $X$-stent. Wall shear-stress $\left(\tau_{w}\right)$ is in Pa.

(Figures 6(d)-6(f) and 7(d)-7(f)). The same sequence of recirculation zone formation, merging, and vortex lift-off was observed at $R e=200$.

\section{B. Flow behavior with and without stent}

The presence of the stent causes significant changes to the near-wall flow behavior, reflected in the change in the distribution of wall shear-stress, with its physiological consequences. This deviation can be examined by comparing the flow field in a stented channel with the flow in an unstented one. There is an analytical solution for the unsteady pulsatile flow in a plane channel $\left(\right.$ Moore $\left.^{16}\right)$, forced by a sinusoidal driving force. The imposed waveform was Fourier-transformed to calculate the exact solution for the present problem; the calculated wall shear-stress was compared with the wall shear-stress distribution obtained from the simulations for $\Lambda$ - and $X$-stents at both Reynolds numbers.

Figures 10 and 11 show the history of the spatially averaged (i.e., averaged over the channel wall) wall shear-stress for $\Lambda$ - and $X$-stents at $R e=80$ and 200, compared with the wall shear-stress in an unstented channel. Several observations can be drawn from these figures. First, we note that when the flow is reversed, the maximum shear is nearly zero, and vice versa for the minimum shear stress. This indicates that the flow is mostly unidirectional in the near-wall region, with high shear in the attached flow regions, and nearly zero stress in the regions of recirculation. For the $X$-stent, the average shear is about half of the maximum one, indicating that the regions of separated flow occupy a significant part of the channel wall; for the $\Lambda$-stent, the difference between spatially averaged and maximum shear is lower, reflecting the smaller extent of the recirculation regions. In the regions of attached flow, the wall shear is close to that in an unstented channel; the maximum shear has a maximum deviation of about $20 \%$ at peak flow rate, for $R e=200$. During the reversed-flow part of the cycle, the agreement is even better. The averaged shear also follows the behavior of the unstented
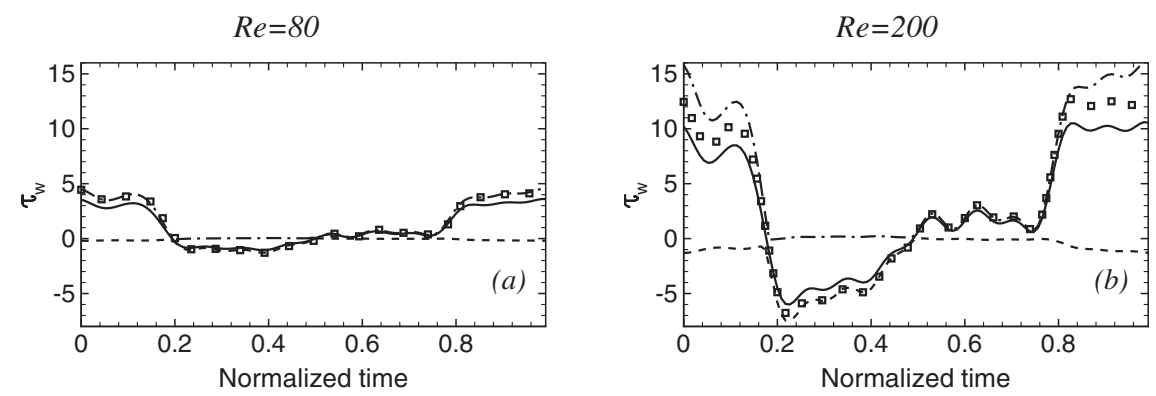

FIG. 11. (a) and (b) History of spatial-averaged (_- ), maximum (-- -), and minimum (- - ) wall shear-stress $\left(\tau_{w}\right)$ and their comparison with wall shear-stress in an unstented channel ( $\square$ ) for the $\Lambda$-stent. Wall shear-stress $\left(\tau_{w}\right)$ is in Pa. 

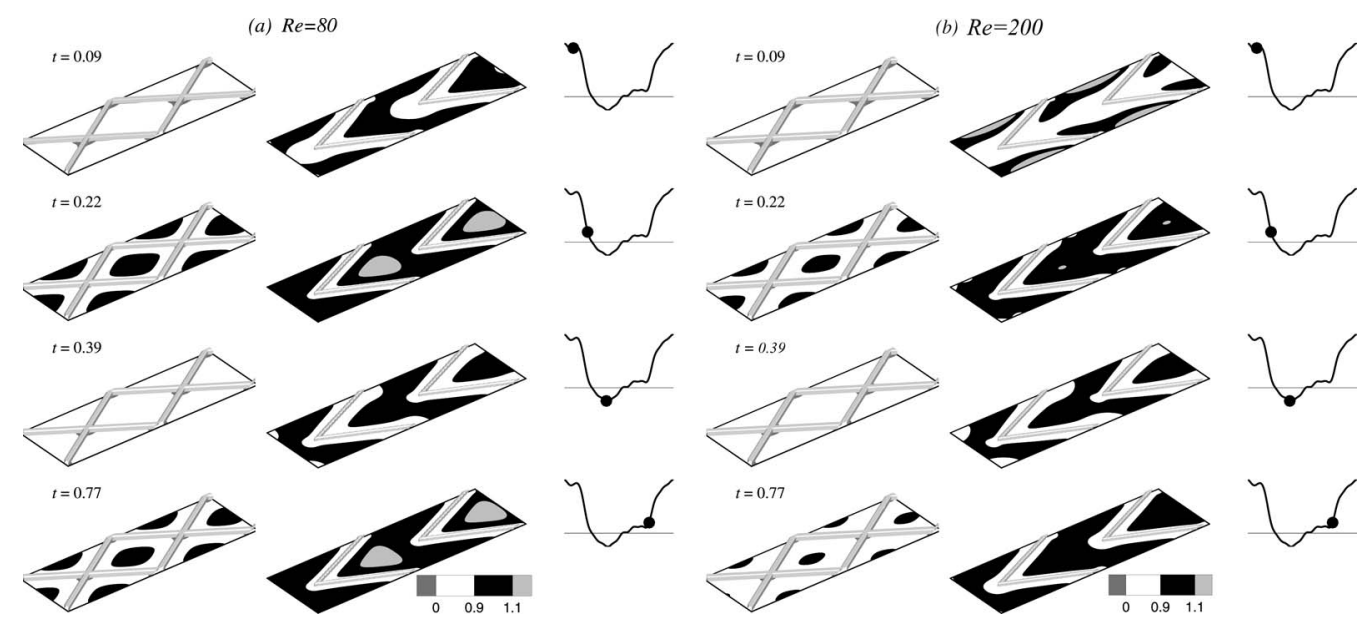

FIG. 12. Area in which wall shear-stress is within $10 \%$ of the one in an unstented channel at (a) $R e=80$ and (b) $R e=200$.

channel, but its magnitude is lower, due to the presence of recirculating flow. In the $\Lambda$-stent, the flow deviates less from the unstented channel and stays mainly attached to the channel wall.

This conclusion is confirmed by Figure 12, which shows contours of the ratio between the wall shear-stress in the stented channel, and the same in the unstented one. Black highlights the regions of the channel wall in which the shear is within $10 \%$ of the unstented-channel value. In the $\Lambda$-stent, over most of the channel the wall stress is equal (or exceeds) the unstented-channel case, both at low and high $R e$. In the $X$-stent, the wall shear (and, hence, the wall stress) is much lower than in a healthy vessel, due to the more extended regions of separated flow. Also note that increasing the Reynolds number increases the significance of advection, and makes the flow in the unstented vessel depart from the exact solution (in which advection is absent). This inference will be justified in Sec. III C.

One notable exception in Figure 12 occurs during the early part of the cycle for the $\Lambda$-stent, when a low-shear region appears downstream of the vertex of the stent wire, which extends to the next stent element when the Reynolds number rises to $R e=200$. This region is due to the generation of two coherent quasi-streamwise vortices that separate and lift up from the stent vertex; these vortices are illustrated in Figure 13. The origin of these vortices is due to the creation of stationary strong vortices covering the stent wires (identified using $Q$ iso-surfaces in Figure 13), which in turn generate these streamwise vortices at the vertex of the stent wire. The upwash region between these vortices appears as a low shear region on the wall. These streamwise vortices were observed at both Reynolds numbers; however, as is shown in Figure 13, they are stronger at $R e=200$ than at $R e=80$, as they remain closer to the wall, contributing more to the wall shear-stress distribution. These vortices were also observed in the $X$-stent (Figure 14) with the same Reynolds number effect and the same origin of formation explained for the $\Lambda$-stent. However, two major differences in these vortices are observed between the $\Lambda$ - and $X$-stents. First, the shape of the $\Lambda$-stent is such that the generated vortices are free on both sides and produce an upwash region in the middle of the channel while downwash regions on the sides while those generated in the $X$-stent are constrained by the stent wires on one side and only produce a downwash region in the middle causing the flow to attach to the wall after separation from the stent strut. Second, the area exposed to these vortices is larger for the $\Lambda$-stent than the $X$-stent. The vortices occupy approximately $28 \%$ of the diamond shape area in the $X$-stent while in the $\Lambda$-stent, $40 \%$ of the area between two subsequent stent wires is occupied by these vortices. Therefore, we expect these vortices to be relatively less important in the near wall flow behavior for the $X$-stent. Also comparing the persisting length of these vortices for different geometries and at the two Reynolds numbers (Figures 13 and 14) indicate that the trajectory of these vortices is more governed by the geometric characteristics of the stent than Reynolds number. 

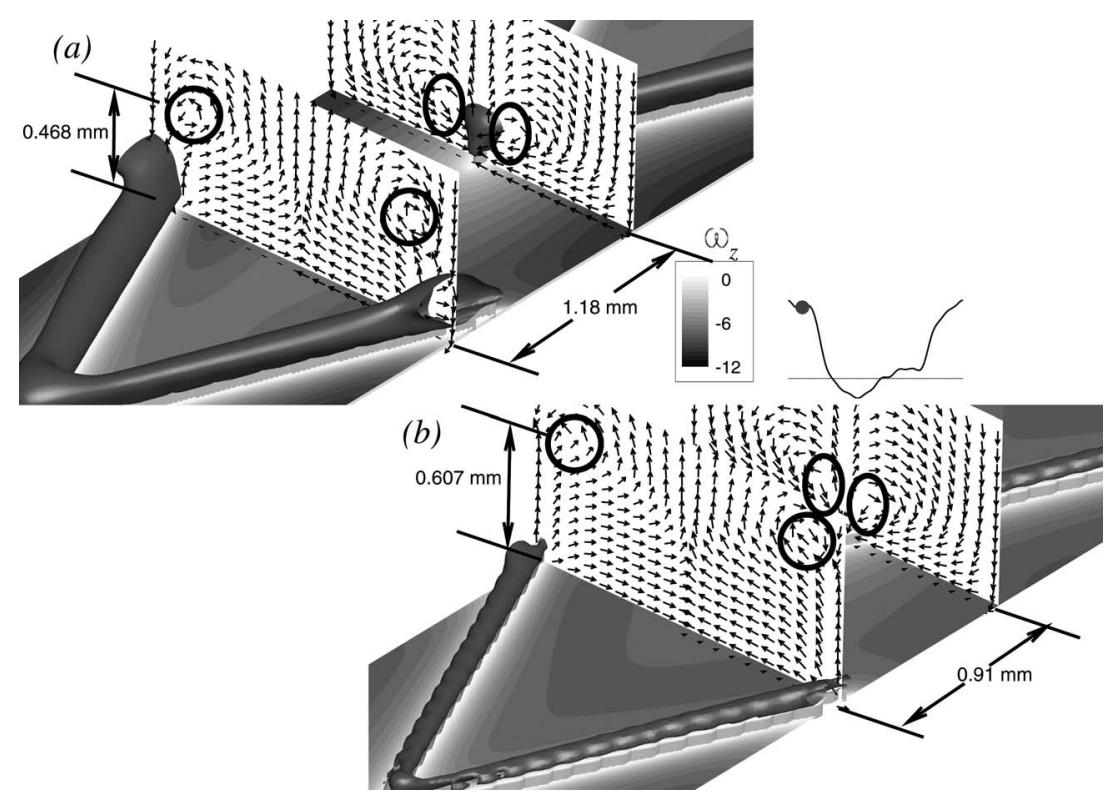

FIG. 13. Velocity vectors in planes normal to the flow, and contours of spanwise vorticity on the wall at $R e=200$ (a) and $R e=80$ (b) for the $\Lambda$-stent. The persisting length of the vortices in addition to the maximum height before their disappearance are indicated.

\section{The role of advection}

At $R e=80$, the Reynolds number based on the stent thickness is approximately 6 , while at $R e=200$ it is approximately 15 . Thus, it can be expected the flow to be dominated by viscous diffusion, and that advection plays a minor role. The similarity between the wall shear in an unstented channel (for which the equations of motion are linear) and the case including the stents suggests that this is indeed the case. If advection effects are negligible, only one calculation would

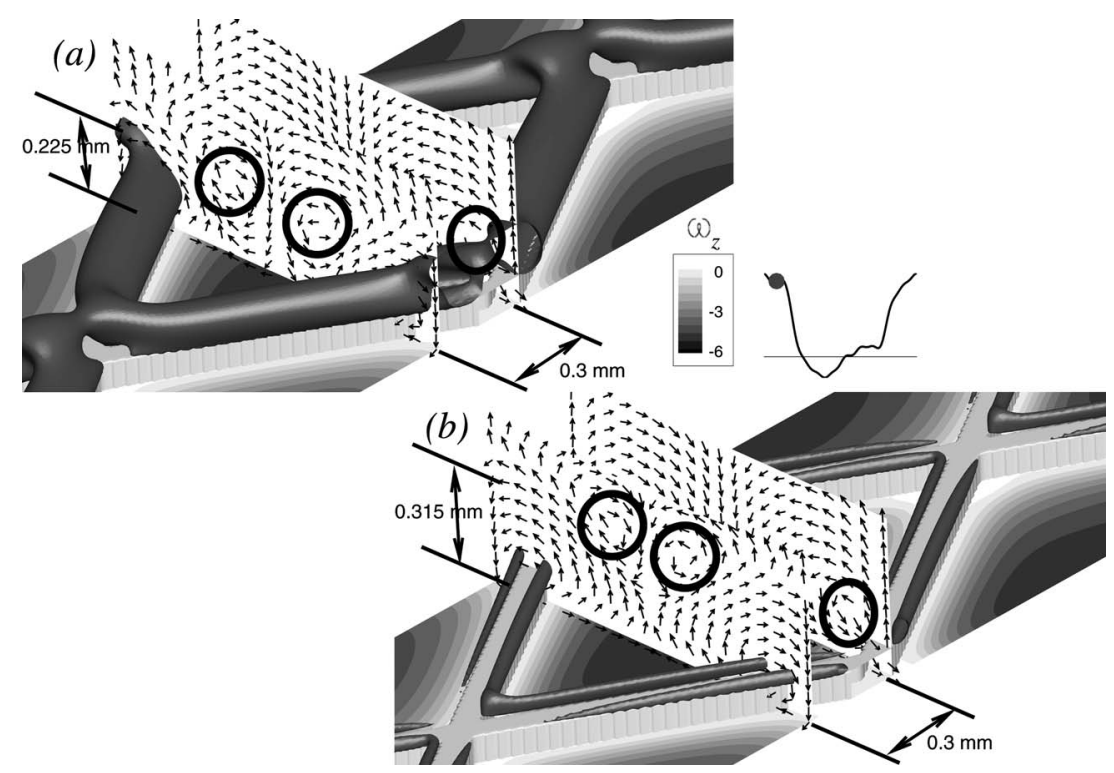

FIG. 14. Velocity vectors in planes normal to the flow, and contours of spanwise vorticity on the wall at $R e=200$ (a) and $R e=80$ (b) for the $X$-stent. The persisting length of the vortices in addition to the maximum height before their disappearance are indicated. 

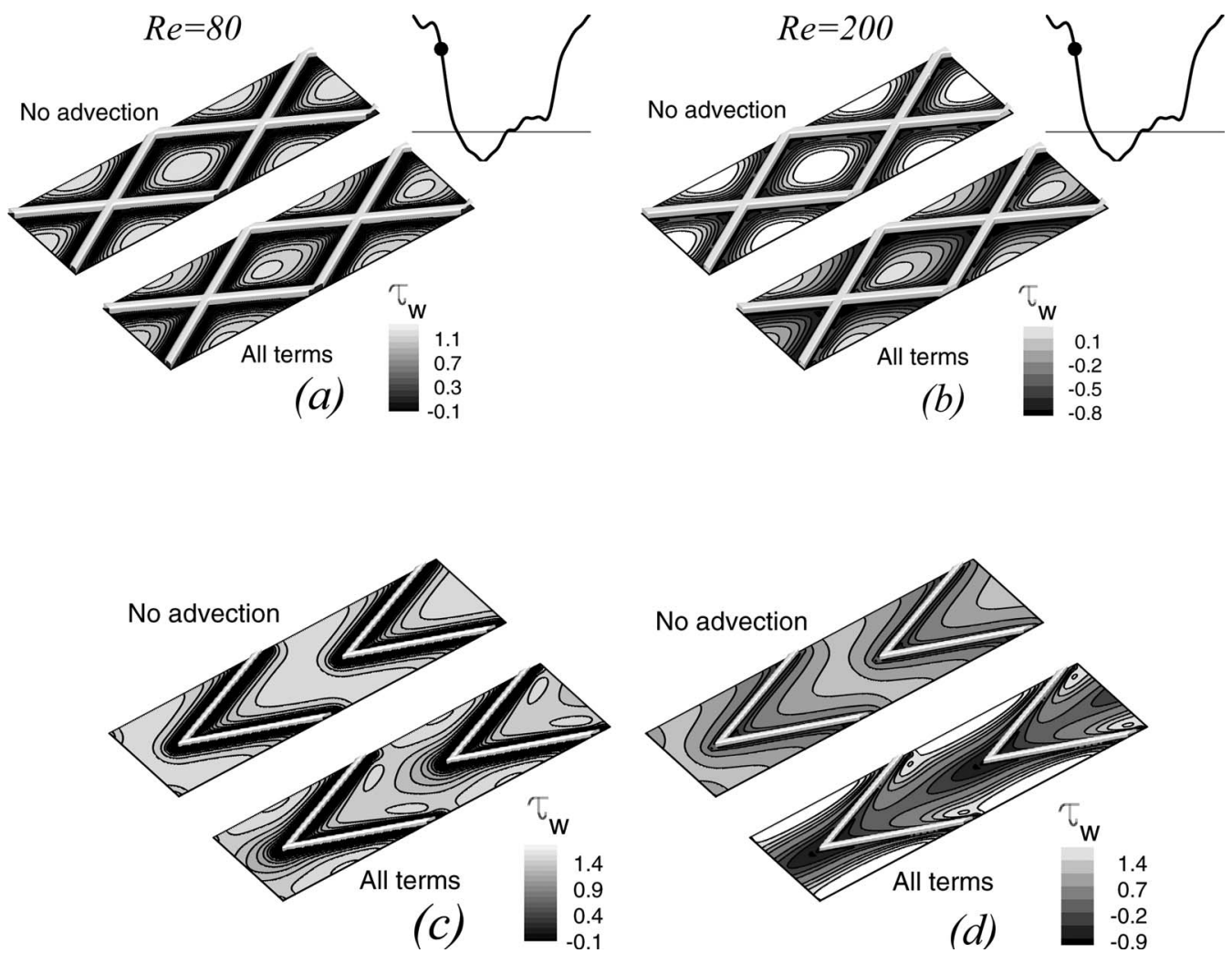

FIG. 15. Comparison of wall shear-stress distribution at (a) and (c) $R e=80$ and (b) and (d) $R e=200$ at the time indicated on the waveforms using the full momentum equation and the one with no advection.

be required for a sinusoidal forcing, and any other waveform could be obtained by superposition of solutions.

To evaluate the effect of the advection terms, we performed simulations in which the advection terms of the momentum equation, $\partial\left(u_{i} u_{j}\right) / \partial x_{j}$, were set to zero; the results are compared with those including all terms in Figure 15, which shows the wall shear-stress at the times at which the most significant effect of advection can be observed. Advective effects are more significant at $R e=200$ (as should be expected). For the $X$-stent, however, their effect is not very large: they modify somewhat the distribution of wall shear, but not its magnitude. In the $\Lambda$-stent, however, their effect is more noticeable even at the lower Re; Figure 16 explains these observations using the dynamics of the streamwise vortices responsible for the upwash mechanism and distribution of wall shear-stress; as can be seen when momentum equations are solved without the advection terms (Figure 16(b)), those streamwise vortices captured in Figure 13, have disappeared. Moreover, the spanwise velocity component is significantly weaker when advection is not considered. Consequently, neglecting the convection terms leads to an even distribution of wall shear-stress (Figure 15) and a significant difference for the $\Lambda$-stent. The disappearance of these vortices was also observed for the $X$-stent when the advection terms were deactivated. However, referring back to Figure 14, it was shown that the streamwise vortices occupy a smaller region of the wetted area for the $X$-stent comparing to the $\Lambda$-stent and on the other hand the effect of advection was reflected as the generation of these vortices. Therefore, a small change in the wall shear-stress distribution for the $X$-stent when no advection exists can be attributed to the small contribution of those streamwise vortices in the near-wall flow behavior for the $X$-stent.

Considering the role of Reynolds number, it was shown in Figures 13 and 14 that the streamwise vortices are playing a more significant role in the near wall region at higher Reynolds number; this explains the importance of advection when Reynolds number is high as was conjectured in Sec. III B. 

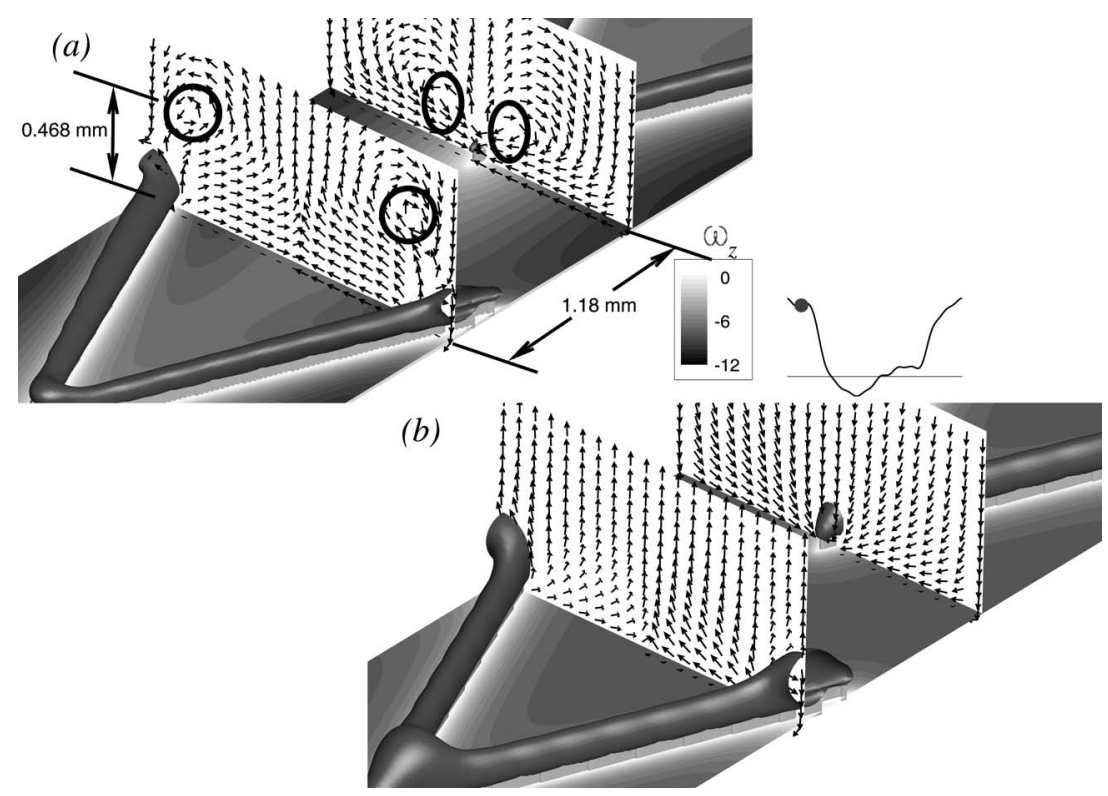

FIG. 16. Velocity vectors in planes normal to the flow, and contours of spanwise vorticity on the wall indicating the importance of advection in formation of streamwise vortices. (a) Flow with all terms and (b) flow without the advection terms.

\section{Vortex creation and migration}

In experimental studies in which reversed flow occurred during part of the period, ${ }^{12-14}$ the creation of large vortical structures and their migration away from the wall was observed. The presence of these vortices leads to low shear stress at the vessel wall, and has biological importance. It was conjectured that the compliance mismatch between the stents and the vessel wall was the cause of this phenomenon. In the present study, however, both the vessel wall and the stent were assumed to be rigid; despite the absence of compliance mismatch, the same vortical structure creation and migration was observed (Figures 6 and 7). The appearance of these vortices appears more related to the characteristics of the oscillating flow. The vortical structures are created from the merging of two recirculation zones that occur during the flow deceleration.

While during a significant part of the cycle an inflection point exists in the velocity profile, the vortices are not formed by a Kelvin-Helmholtz-like instability mechanism; in fact, the center of the vortex core (as visualized from contours of $Q$ ) occurs well below the inflection point through most of the cycle, and moves above it only late, when the vortex is advected toward the centerline.

The driving waveform plays a significant role in the creation of the vortex, which appears toward the end of the deceleration stage. Starting from the waveform used by Charonko et al., ${ }^{13,14}$ we gradually increased the mean flowrate (thereby decreasing the period over which the flow was reversed, Figure 17). This leads to an increase of the Reynolds number from 80 to 175 for the low-Re case, and from 200 to 450 for the high-Re one. The figure shows the times at which vortices are
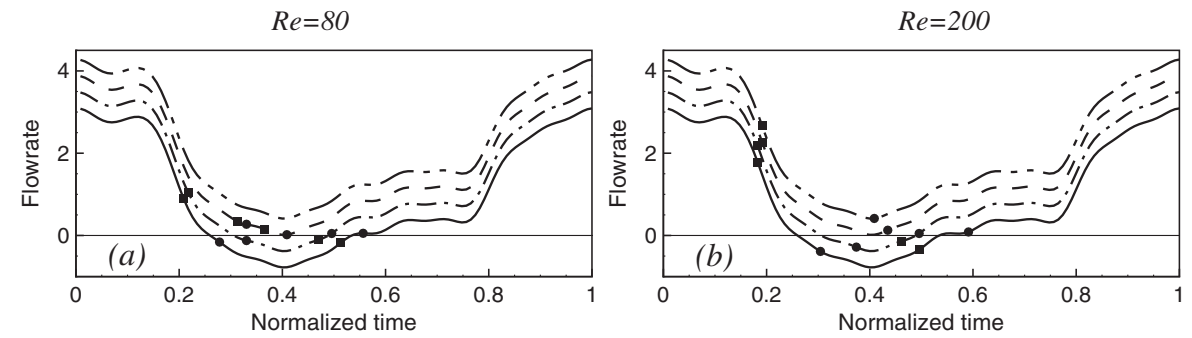

FIG. 17. (a) and (b) Times at which vortices are created (

) and disappear $(\bullet)$ for different waveforms in the $\Lambda$-stent. 

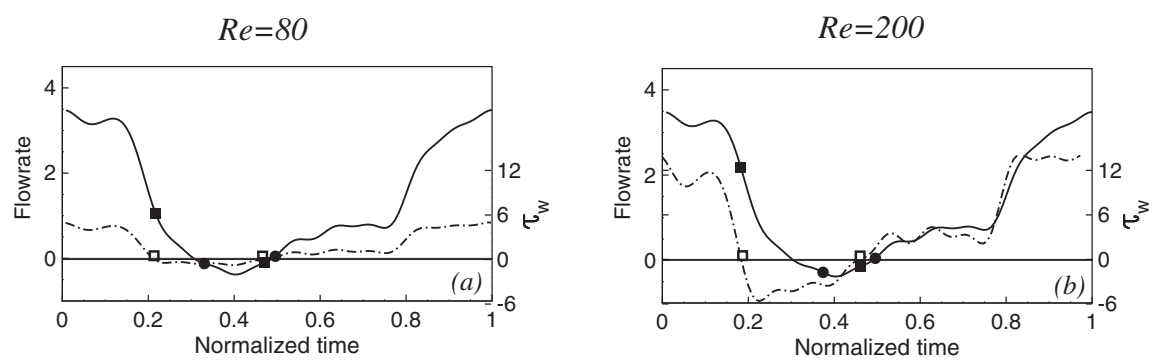

FIG. 18. Times at which vortices are created $(\boldsymbol{\square})$ and disappear $(\bullet)$ for the driving waveform on stented channels $(-)$ with (a) base $R e=80$ and (b) base $R e=200$. History of wall shear-stress (-.-) and times at which wall shear-stress changes its sign ( $\square$ ). Wall shear-stress $\left(\tau_{w}\right)$ is in Pa.

created ( $\mathbf{\bullet})$ and disappear $(\bullet)$. First, these times are the same for both $\Lambda$ - and $X$-stents: they seem to be independent of the stent geometry and only depend on the imposed flow condition (Reynolds number and waveform). Vortex formation is observed even in cases in which no mean-flow reversal occurs. The flow may reverse in the near-wall region even if the mean flow is unidirectional, and in fact we observe that the appearance of the vortices coincides with the appearance of near-wall flow reversal (Figure 18).

\section{CONCLUSIONS}

An immersed-boundary method was used to perform numerical simulation of two idealized stents, inspired by actual geometries; the same waveform used by Charonko et al. ${ }^{13,14}$ was applied. The numerical results agree very well with the experimental ones qualitatively and quantitatively, if the geometric differences are taken into account.

Vortex creation and migration was observed, consistent with the experimental findings by Yazdani et al. ${ }^{12}$ and Charonko et al. ${ }^{13,14}$ It had been previously conjectured that the vortex formation was caused by the compliance mismatch between the vessel wall and the stents. The key finding in this study is that, in spite of the absence of compliance, and by employing a rigid stent and vessel, this vortex formation and migration was observed, in contrast to the previous presumption. The presence of the stent wires constrains the flow and causes the reversal to result in the formation of one or two recirculation zones (depending on the stent geometry). During the deceleration phase, these recirculation zones merge, giving rise to a large, vortical structure that migrates toward the channel center.

In the $\Lambda$-stent, the flow remained attached to the wall during most of the cycle, while the large circulation zones created due to the $X$-stent caused the flow to differ significantly from that in an unstented channel. Increasing the Reynolds number resulted in further deviation from the unstented channel behavior, for both geometries. Study of the flow field revealed the formation of two quasistreamwise vortices for both stents and at both Reynolds numbers which more significantly altered the wall stress distribution for the $\Lambda$-stent, especially in the upwash region between the stent wires.

At $R e=80$, the flow is diffusion dominated, and neglecting the advection terms in the NavierStokes equations did not result in significant changes in the flow field; at $R e=200$ both advection and diffusion are important. The formation of the quasi-streamwise vortices mentioned above is a nonlinear phenomenon that was observed only when the advection terms were included; this correlation found between these vortices and advection could explain the importance of advection for the two stents and at the two Reynolds numbers. These vortices were closer to the wall at higher Reynolds number contributing more to the wall shear-stress distribution and signifying the role of advection. Also since the exposed area of the channel under these vortices was larger for the $\Lambda$-stent comparing to the $X$-stent, the role of advection for the $\Lambda$-stent was more significant than the $X$-stent.

While this study indicates that geometries similar to the $\Lambda$-stent may present a lower risk of vessel blockage (as the area of the recirculation zones is reduced) several questions remain open. More realistic problem setups including the motion of the vessel wall, the non-Newtonian behavior 
of the flow, and the tracking of biological particles and their accumulation on the vessel wall are needed to improve the understanding of the fluid-dynamical behavior of stents. Furthermore, the geometric details (curvature of the stent wires, presence of connectors, etc.) have not been considered here. Preliminary results (Figure 5) indicate that, while they do not affect the global features of the flow, they may change the wall stress distribution and average values by $29 \%$. An investigation of this issue is ongoing.

\section{ACKNOWLEDGMENTS}

We would like to thank Dr. Matthew David Ford for performing the calculations on OpenFOAM and for his valuable comments on the results. U.P. and A.R. were partially supported by the National Science and Engineering Research Council (NSERC). U.P. also acknowledges the support of Canada Research Chairs program. We also thank the High Performance Computing and Virtual Laboratory (HPCVL), Queen's University site (www.hpcvl.org), for the computational support.

${ }^{1}$ D. P. Hajjar and A. C. Nicholson, "Atherosclerosis-cellular and molecular mechanisms cause hardening of the arteries," Am. Sci. 83, 460-467 (1995), http://www.jstar.org/stable/29775523.

${ }^{2}$ M. S. Chen, J. M. John, D. P. Chew, D. S. Lee, S. G. Ellis, and D. L. Bhatt, "Bare metal stent restenosis is not a benign clinical entity," Am. Heart J. 151, 1260-1264 (2006).

${ }^{3}$ J. Murphy and F. Boyle, "Predicting neointimal hyperplasia in stented arteries using time-dependant computational fluid dynamics: A review," Comput. Biol. Med. 40, 408-418 (2010).

${ }^{4}$ D. Bluestein, L. Niu, R. T. Schoephoerster, and M. K. Dewanjee, "Fluid mechanics of arterial stenosis: Relationship to the development of mural thrombus," Biomed. Eng. Soc. 25, 344-356 (1997).

${ }^{5}$ C. G. Caro, J. M. Fitz-Gerald, and R. C. Schroter, "Atheroma and arterial wall shear: Observation, correlation and proposal of a shear dependent mass transfer mechanism for atherogenesis," Proc. R. Soc. London 177, 109-159 (1971).

${ }^{6}$ D. L. Fry, "Certain histological and chemical responses of the vascular interface to acutely induced mechanical stress in the aorta of the dog," Circ. Res. 24, 93-108 (1969).

${ }^{7}$ C. K. Zarins, D. P. Giddens, B. K. Bharadvaj, V. S. Sottiurai, R. F. Mabon, and S. Glagov, "Carotid bifurcation atherosclerosis: Quantitative correlation of plaque localization with flow velocity profiles and wall shear stress," Circ. Res. 53, 502-514 (1983).

${ }^{8}$ A. M. Malek, S. L. Alper, and S. Izumo, "Hemodynamic shear stress and its role in atherosclerosis," J. Am. Med. Assoc. 282(21), 2035-2042 (1999)

${ }^{9}$ J. J. Wentzel, R. Krams, J. C. H. Schuurbiers, J. A. Oomen, J. Kloet, W. J. van der Giessen, P. W. Serruys, and C. J. Slager, "Relationship between neointimal thickness and shear stress after wallstent implantation in human coronary arteries," Circulation 103, 1740-1745 (2001).

${ }^{10}$ N. Duraiswamy, B. Jayachandran, J. Byrne, J. E. Moore, and R. T. Schoephoerster, "Spatial distribution of platelet deposition in stented arterial models under physiologic flow," Ann. Biomed. Eng. 33, 1767-1777 (2005).

${ }^{11}$ N. Duraiswamy, J. M. Cesar, R. T. Schoephoerster, and J. E. Moore, "Effects of stent geometry on local flow dynamics and resulting platelet deposition in an in vitro model," Biorheology 45, 547-561 (2008).

${ }^{12}$ S. K. Yazdani, Jr., J. E. Mooore, J. L. Berry, and P. Vlachos, "DPIV measurements of low disturbances in stented artery models: Adverse affects of compliance mismatch," J. Biomech. Eng. 126, 559-566 (2004).

${ }^{13}$ J. Charonko, S. Karri, J. Schmieg, S. Prabhu, and P. Vlachos, "In vitro, time-resolved PIV comparison of the effect of stent design on wall shear stress," Ann. Biomed. Eng. 37, 1310-1321 (2009).

${ }^{14}$ J. Charonko, S. Karri, J. Schmieg, S. Prabhu, and P. Vlachos, "In vitro comparison of the effect of stent configuration on wall shear stress using time-resolved particle image velocimetry," Ann. Biomed. Eng. 38, 889-902 (2010).

${ }^{15}$ J. L. Berry, A. Santamarina, Jr., J. E. Moore, S. Roychowdhury, and W. D. Routh, "Experimental and computational flow evaluation of coronary stents," Ann. Biomed. Eng. 28, 386-398 (2000).

${ }^{16}$ F. K. Moore, Theory of Laminar Flows (Oxford University Press, 1964).

${ }^{17}$ J. F. LaDisa, Jr., I. Guler, L. E. Olson, D. A. Hettrick, J. R. Kersten, D. C. Warltier, and P. S. Pagel, "Three-dimensional computational fluid dynamics modeling of alterations in coronary wall shear stress produced by stent implantation," Ann. Biomed. Eng. 31, 972-980 (2003).

${ }^{18}$ J. F. LaDisa, Jr., L. E. Olson, I. Guler, D. A. Hettrick, S. H. Audi, J. R. Kersten, D. C. Warltier, and P. S. Pagel, "Stent design properties and deployment ratio influence indexes of wall shear stress: A three-dimensional computational fluid dynamics investigation within a normal artery," J. Appl. Physiol. 97, 424-430 (2004).

${ }^{19}$ J. F. LaDisa, Jr., L. E. Olson, I. Guler, D. A. Hettrick, J. R. Kersten, D. C. Warltier, and P. S. Pagel, "Circumferential vascular deformation after stent implantation alters wall shear stress evaluated with time-dependent 3D computational fluid dynamics models," J. Appl. Physiol. 98, 947-957 (2005).

${ }^{20}$ D. N. Ku, "Blood flow in arteries," Annu. Rev. Fluid Mech. 29, 399-434 (1997).

${ }^{21}$ J. F. LaDisa, Jr., L. E. Olson, D. A. Hettrick, D. C. Warltier, J. R. Kersten, and P. S. Pagel, "Alterations in regional vascular geometry produced by theoretical stent implantation influence distributions of wall shear stress: Analysis of a curved coronary artery using 3D computational fluid dynamics modeling," Biomed. Eng. Online 5, 40-50 (2006).

${ }^{22}$ R. Glenny, W. Lamm, R. Albert, and H. Robertson, "Gravity is a minor determinant of pulmonary blood flow distribution," J. Appl. Physiol. 71, 620-629 (1991). 
${ }^{23}$ R. Glenny, L. Polissar, and H. Robertson, "Relative contribution of gravity to pulmonary perfusion heterogeneity,” J. Appl. Physiol. 71, 2449-2452 (1991).

${ }^{24}$ A. Keating, U. Piomelli, K. Bremhorst, and S. Nei, "Large-eddy simulation of heat transfer downstream of a backwardfacing step," J. Turbul. 5, 1-27 (2004).

${ }^{25}$ T. Seo, L. G. Schachter, and A. I. Barakakt, "Computational study of fluid mechanical disturbance induced by endovascular stents," Ann. Biomed. Eng. 33, 444-456 (2005).

${ }^{26}$ A. J. Chorin, "On the convergence of discrete approximations to the Navier-Stokes equations," Math. Comput. 23, 341-353 (1969).

${ }^{27}$ J. Kim and P. Moin, "Application of a fractional-step method to incompressible Navier- Stokes equations," J. Comput. Phys. 59, 308-323 (1985)

${ }^{28}$ C. W. Hirt and B. D. Nichols, "Volume of fluid (VOF) method for the dynamics of free boundaries," J. Comput. Phys. 39, 201-225 (1981).

${ }^{29}$ H. Weller, G. Tabor, H. Jasak, and C. Fureby, "A tensorial approach to computational continuum mechanics using object-oriented techniques," Comput. Phys. 12, 620-631 (1998).

${ }^{30}$ B. F. Armaly, F. Durst, J. C. F. Pereira, and B. Schönung, "Experimental and theoretical investigation of backward-facing step flow," J. Fluid Mech. 127, 473-496 (1983). 